\title{
Minimal surfaces in porous media: Pore-scale imaging of multiphase flow in an altered-wettability Bentheimer sandstone
}

\author{
Qingyang Lin, ${ }^{1,}$ Branko Bijeljic, ${ }^{1}$ Steffen Berg, ${ }^{2}$ Ronny Pini, ${ }^{3}$ Martin J. Blunt, ${ }^{1}$ and Samuel Krevor ${ }^{1}$ \\ ${ }^{1}$ Department of Earth Science and Engineering, Imperial College London, London, SW7 2AZ, United Kingdom \\ ${ }^{2}$ Shell Global Solutions International B.V., Grasweg 31, $1031 \mathrm{HW}$, Amsterdam, The Netherlands \\ ${ }^{3}$ Department of Chemical Engineering, Imperial College London, London, SW7 2AZ, United Kingdom
}

(Received 11 March 2019; published 10 June 2019)

\begin{abstract}
High-resolution x-ray imaging was used in combination with differential pressure measurements to measure relative permeability and capillary pressure simultaneously during a steady-state waterflood experiment on a sample of Bentheimer sandstone $51.6 \mathrm{~mm}$ long and $6.1 \mathrm{~mm}$ in diameter. After prolonged contact with crude oil to alter the surface wettability, a refined oil and formation brine were injected through the sample at a fixed total flow rate but in a sequence of increasing brine fractional flows. When the pressure across the system stabilized, $\mathrm{x}$-ray tomographic images were taken. The images were used to compute saturation, interfacial area, curvature, and contact angle. From this information relative permeability and capillary pressure were determined as functions of saturation. We compare our results with a previously published experiment under water-wet conditions. The oil relative permeability was lower than in the water-wet case, although a smaller residual oil saturation, of approximately 0.11 , was obtained, since the oil remained connected in layers in the altered wettability rock. The capillary pressure was slightly negative and 10 times smaller in magnitude than for the water-wet rock, and approximately constant over a wide range of intermediate saturation. The oil-brine interfacial area was also largely constant in this saturation range. The measured static contact angles had an average of $80^{\circ}$ with a standard deviation of $17^{\circ}$. We observed that the oil-brine interfaces were not flat, as may be expected for a very low mean curvature, but had two approximately equal, but opposite, curvatures in orthogonal directions. These interfaces were approximately minimal surfaces, which implies well-connected phases. Saddle-shaped menisci swept through the pore space at a constant capillary pressure and with an almost fixed area, removing most of the oil.
\end{abstract}

DOI: 10.1103/PhysRevE.99.063105

\section{INTRODUCTION}

High-resolution x-ray imaging has led to transformational improvements in our understanding of multiphase flow in porous media, enabling the visualization of pore-scale displacement processes under reservoir conditions of high temperature and pressure [1,2]. This work has been used to address a wide range of topics, including the dynamics of drainage and imbibition [3-8], contact angle distributions [9-13], interfacial area [14], and interfacial curvature [15-20].

While this research has led to a much improved understanding of fluid flow, it has hitherto been somewhat disconnected from the traditional core-scale measurements of relative permeability and capillary pressure that are used to describe multiphase flow. One of the key parameters that controls the relative permeability and capillary pressure relationships is the wetting state of the rock. A common procedure in special core analysis is to restore the wettability state of the reservoir, which can be altered from a mainly hydrophilic state of most minerals, to a more mixed-wet condition due to adsorption of surface active crude oil components by reintroducing the reservoir fluids to reproduce the pore-scale fluid configuration present in the reservoir [21].

*Corresponding author: q.lin11@imperial.ac.uk
We propose to combine measurements of multiphase flow properties with pore-scale imaging, an approach that offers many advantages over current methods to characterize rock samples. First, the imaging experiments are usually performed on smaller samples than those in traditional analysis. This enables homogeneous samples to be selected [22], removing the complexities of centimeter-scale heterogeneity on the behavior [23], and the experiments take less time, as capillary equilibrium is established more quickly. The effects of largerscale heterogeneity can be accounted for once the small-scale flow properties are determined, again using a combination of flow experimentation and imaging [24-26]. Furthermore, from estimations of interfacial curvature, capillary pressure can be found [17] simultaneously with relative permeability [20], which is challenging to achieve with conventional methods. Last, the images themselves provide insight into displacement processes, can be used to correct for the capillary end effect [20], and provide benchmark data to test and validate pore-scale models [27].

Gao et al. [28] measured the steady-state waterflood relative permeability for a water-wet Bentheimer sample. They investigated the effects of flow rate to show the onset of intermittent pore occupancy, when some regions of the pore space are alternately occupied by oil and water [8]. Lin et al. [20] further extended the methodology and measured capillary pressure, from interfacial curvature, as well as relative 
permeability, again from a steady-state experiment on waterwet Bentheimer sandstone. A gradient in saturation and capillary pressure was observed in the flow direction, and this effect-the capillary end effect-was corrected for analytically in the measurement of relative permeability. Zou et al. [29] measured the steady-state oil-injection relative permeability in a sample of Bentheimer that had been treated with surface-active agents and compared the results with a similar water-wet system.

Furthermore, high-resolution imaging offers the ability to observe new phenomena. Specifically, to date our conceptualization of multiphase flow has been based around quasitwo-dimensional experiments in micromodels [30], simulations (see, for instance, Ref. [31]), and theoretical analysis [32]. Hence it has hitherto been assumed that the fluidfluid menisci either are curved in only one direction or are approximately hemispherical with two approximately equal curvatures. While this has been seen in water-wet media [15], for mixed-wet media the unchallenged assumption has been that menisci would change smoothly from spherical interfaces with oil bulging into water (positive capillary pressure) to water bulging into oil (a negative capillary pressure) with the unstated implication that a flat interface would be seen at the transition from positive to negative capillary pressures. Despite the huge interest in other scientific communities in interfaces with curvatures of different sign in orthogonal directions [33], this possibility had been overlooked in the context of porous media flow. As we demonstrate in this paper, in fact such interfaces are likely to be universal in natural systems.

Therefore, the aims of this work are twofold: first, to measure relative permeability and capillary pressure during a steady-state waterflooding in a rock sample whose wettability has been altered through contact with crude oil (called aging) following the wettability restoration practice from special core analysis. We will use our pore-space images to quantify interfacial areas, curvatures, and contact angles and to explain the pore-scale physics that control the measured macroscopic flow properties. Second, we will use our images to uncover the existence of interfaces that are, approximately, minimal surfaces with a mean curvature close to zero, but with equal and opposite curvatures in orthogonal directions.

\section{METHODOLOGY}

The rock sample we used in this study was Bentheimer sandstone with an average porosity of 0.24 (measured with Autopore 9520, Micromeritics). Cylindrical rock samples called cores with $6.1 \mathrm{~mm}$ diameter and length of approximately $26 \mathrm{~mm}$ were drilled from a larger cylinder of rock (38 mm diameter and $40 \mathrm{~mm}$ long). The larger sample containing predrilled small cores was then fully saturated with potassium iodide (KI) doped brine (see

TABLE I. Brine composition.

\begin{tabular}{lccc}
\hline \hline $\mathrm{N}_{\mathrm{a} l} \mathrm{Cl}$ & $\mathrm{C}_{\mathrm{a}} \mathrm{Cl}_{2} .2 \mathrm{H}_{2} \mathrm{O}$ & $\mathrm{M}_{\mathrm{g}} \mathrm{Cl}_{2} .6 \mathrm{H}_{2} \mathrm{O}$ & $\mathrm{KI}$ \\
\hline $1.09 \mathrm{wt} \%$ & $0.11 \mathrm{wt} \%$ & $0.02 \mathrm{wt} \%$ & $3.5 \mathrm{wt} \%$ \\
\hline \hline
\end{tabular}

Table I for the brine composition; the viscosity of the brine was $1.01 \pm 0.02 \mathrm{mPa} \cdot \mathrm{s}$ at $20^{\circ} \mathrm{C}$ ), followed by centrifugation (equivalent capillary pressure of $20 \mathrm{kPa}$ ) with crude oil to initialize the sample with a brine saturation $\left(S_{w i}\right)$ of approximately $10 \%$ [20]. The crude oil used in this study came from a producing field in the Middle East, supplied by Shell Global Solutions International BV (TAN $=0.09 \mathrm{mgKOH} / \mathrm{g}$, TBN $=0.270 \mathrm{mg} / \mathrm{g}$, SARA analysis: saturates $=44.0$, aromates $=44.0$, resins $=9.69$, asphaltenes $=2.31$, density $=850 \mathrm{~kg} / \mathrm{m}^{3}$, viscosity $=$ $8-10 \mathrm{mPa} \cdot \mathrm{s}$ at room temperature). This assembly with multiple small cores was submerged in the same crude oil at 3 MPa pressure and $80^{\circ} \mathrm{C}$ for 4 weeks to alter the wettability. The details of this sample preparation protocol can be found in our previous study [34].

\section{A. Apparatus and experimental procedure}

Steady-state conditions were reached in sequence through injecting at different fractional flows $\left(f_{w}=0,0.02,0.06\right.$, $0.24,0.5,0.8,0.9,1$ ) with a total flow rate of $0.03 \mathrm{ml} / \mathrm{min}$ (the total Darcy velocity was $1.7 \times 10^{-5} \mathrm{~m} / \mathrm{s}$ ). The fractional flow is defined as the volumetric injection rate of brine divided by the total rate of brine and oil. The injection occurred over a period of at least $12 \mathrm{~h}$ for each fractional flow step. The injection time for each fractional flow varied: we waited until the differential pressure was constant over a period of at least $3 \mathrm{~h}$. The maximum capillary number $(\mathrm{Ca}=\mu q / \sigma$, where $q$ is the Darcy velocity of the invading fluid (brine), $\mu$ is is the brine viscosity, and $\sigma$ is the brine-oil interfacial tension) was $9.9 \times 10^{-7}$ when the fractional flow $f_{w}=1$. Decalin (cis- and trans-decahydronaphthalene mixture with a dynamic viscosity of $2.96 \pm 0.08 \mathrm{mPa} \cdot \mathrm{s}$ at $20^{\circ} \mathrm{C}$, supplied by Alfa Aesar) was selected as the oil phase. The interfacial tension between brine and decalin was measured to be $51.5 \pm 1.6 \mathrm{mN} / \mathrm{m}$, at ambient conditions measured by the pendant drop method (Rame-Hart $590 \mathrm{~F} 4$ series) $[35,36]$. The experimental apparatus is shown in Fig. 1. The experimental procedure was as follows:

1. Two rock samples $(6.1 \mathrm{~mm}$ diameter with a total length of $51.6 \mathrm{~mm}$ ) that had been saturated with crude oil and formation brine (aged) were taken and loaded in series into the core holder in the presence of the oil phase (decalin) to prevent air entering the system.

2. A confining pressure of $2 \mathrm{MPa}$ was applied and maintained within the cell to compress the Viton sleeve around the samples to avoid fluid bypass.

3. The system was then pressurized (line pressure of 5 $\mathrm{MPa}$ ), and 1000 pore volumes of the oil phase was injected to replace the crude oil in the sample.

4. The system was then depressurized to ambient pressure with a 3 MPa back pressure. The experiments were performed at ambient temperature (approximately $20^{\circ} \mathrm{C}$ ).

5. The steady-state waterflood experiment started with injection of the oil phase at a flow rate of $0.03 \mathrm{ml} / \mathrm{min}$ $\left(f_{w}=0\right)$. After reaching steady state, which was indicated by a constant differential pressure transducer measurement, scans were taken to obtain the initial brine saturation. Each scan took approximately $1 \mathrm{~h}$ to complete. Three scans were taken over the middle section of the downstream sample covering a vertical distance of $10.7 \mathrm{~mm}$ with a voxel size of $3.58 \mu \mathrm{m}$. The 


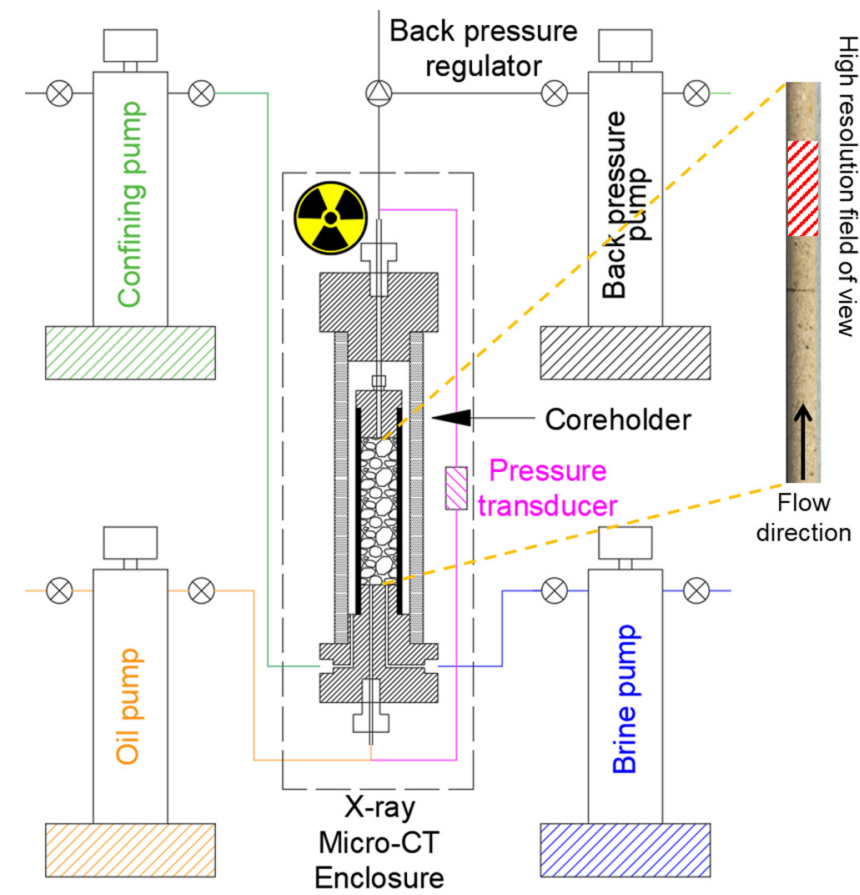

FIG. 1. Schematic diagram of the experimental apparatus using a Hassler-type flow cell made of carbon fiber epoxy with low x-ray photon attenuation. Tomographic scans were taken for the middle section of the downstream sample over a vertical distance of $10.7 \mathrm{~mm}$ with a voxel size of $3.58 \mu \mathrm{m}$.

differential transducer used in this study was a Keller PD-33X, with an accuracy of $\pm 0.3 \mathrm{kPa}$.

6. Brine and oil were both injected at the same time at $f_{w}=0.02$, with a total flow rate of $0.03 \mathrm{ml} / \mathrm{min}$. Injection continued until steady state was achieved indicated by a stable pressure differential. Then scans were taken as described in step 5.

7. Step 6 was repeated with different fractional flows $f_{w}=0.02,0.06,0.24,0.5,0.8,0.9,1$ while keeping the total volumetric flow rate constant at $0.03 \mathrm{ml} / \mathrm{min}$. Scans were taken once steady state had been reached for each fractional flow.

8. Steps 5-7 were also repeated without a sample inside the core holder to record the pressure drop due to the flow resistance of the core holder and flow lines alone.

9. After the experiment, the samples were cleaned with toluene using the soxhlet extraction method.

10. The cleaned samples were then loaded again with a 2 MPa confining pressure.

11. Scans of the air-saturated rocks were taken at room temperature.

12. Gaseous $\mathrm{CO}_{2}$ was injected for $30 \mathrm{~min}$ to remove air.

13. The rocks were flooded with brine (doped with 30 wt $\% \mathrm{KI}$ ). After $100 \%$ brine saturation was achieved, scans were taken of the brine-saturated rock sample to characterize the porosity distribution including porosity below the image resolution using differential imaging. The detailed procedure can be found in Lin et al. [37]. This allows the brine saturation to be accurately calculated.
14. The rocks were then flooded with brine (see the brine composition in Table I) again for 1000 pore volumes to replace the high-salinity brine in the previous step.

15. The absolute permeability of the sample was then measured from the pressure differential at a known flow rate when fully saturated with a single phase (brine).

Finally, the experiment was repeated on another sample that had undergone an identical aging protocol. However, we studied only a single fractional flow step $f_{w}=0.5$. The purpose was to determine if the surprising interfacial curvatures measured were reproducible.

The apparatus was similar to that used in our previous studies $[20,28]$. There was a specially designed inlet end piece which allowed both phases to enter the sample simultaneously. Due to the high permeability of Bentheimer sandstone, two cores were assembled in series (51.6 $\mathrm{mm}$ in total length) to enhance the pressure signal and measure an accurate pressure differential. The absolute permeability, measured at the same experimental conditions, was $2.17 \pm 0.05 \times 10^{-12} \mathrm{~m}^{-2}$ obtained in step 15 in the procedure above.

\section{B. Image acquisition, image processing, and segmentation}

The imaging was performed using a Zeiss Versa $510 \mathrm{x}$-ray microscope with a flat panel detector. The voxel size of the images was $3.58 \mu \mathrm{m}$. At each steady-state point with different fractional flows, three scans (while flowing) were captured and stitched to a larger field of view (10.7 mm in length).

All the images were registered to have the same orientation, followed by applying a nonlocal means edge preserving filter [38] for noise reduction. The filtered images were segmented into three phases (grain, brine, and oil) using a

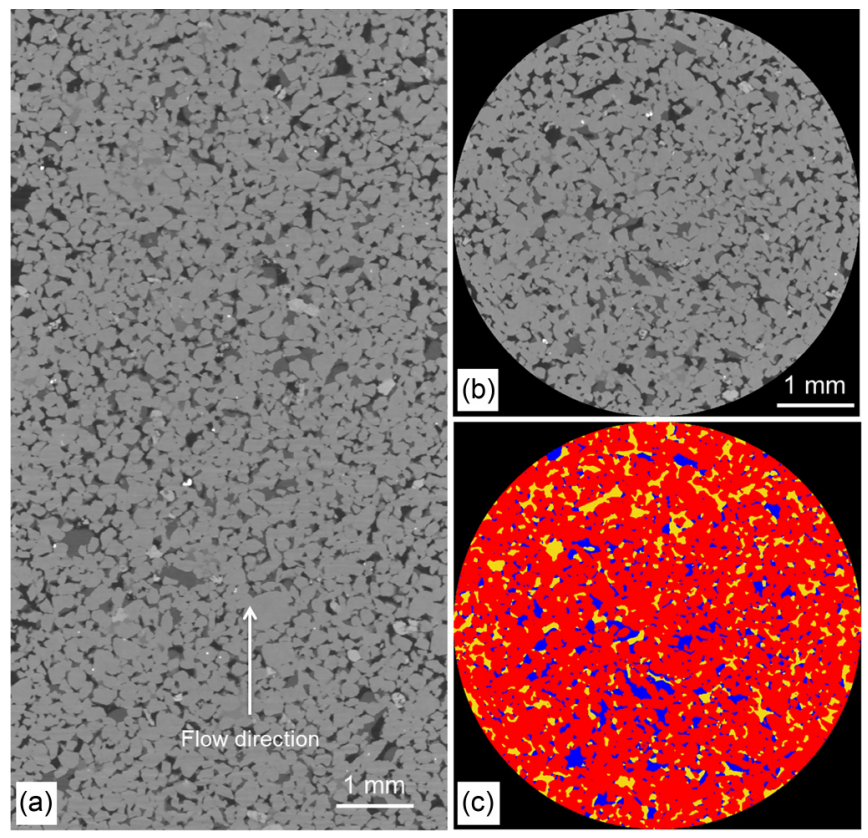

FIG. 2. (a),(b) Gray-scale two-dimensional cross sections of a three-dimensional image at $f_{w}=0.5$ after applying a nonlocal means edge-preserving filter. The dimension of the image is $1600 \times 1600 \times$ 3000 voxels with $3.58 \mu \mathrm{m}$. (c) Three-phase segmentation showing rock grains (red), brine (blue), and oil (yellow). 


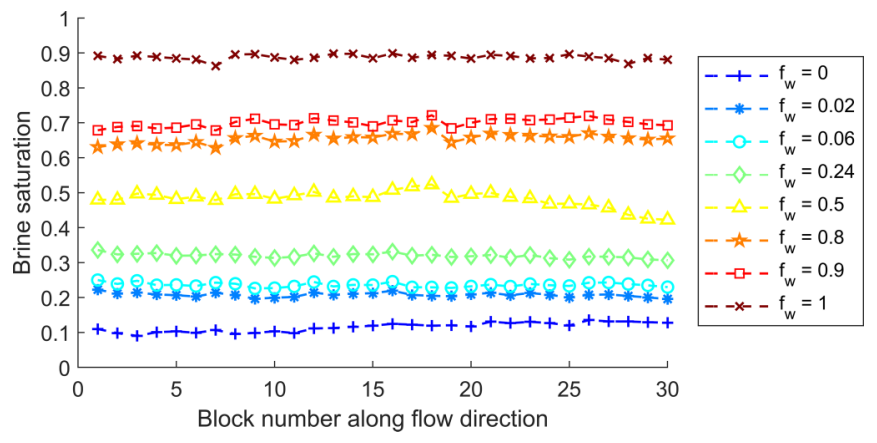

FIG. 3. The saturation averaged in each block along the direction of flow. Each block contains $1600 \times 1600 \times 100$ voxels (approximately $0.36 \mathrm{~mm}$ in length along the sample). The saturation is computed using the total porosity of 0.231 from the differential imaging method.

seeded watershed algorithm [15,39]. The image processing and segmentation were consistent with our previous study on water-wet Bentheimer samples [20]. The image dimension of the entire field of view is $1600 \times 1600 \times 3000$ voxels with a $3.58 \mu \mathrm{m}$ voxel size: see Fig. 2 for example images.

\section{IMAGE-BASED CHARACTERIZATION OF MULTIPHASE FLOW PROPERTIES DURING STEADY STATE}

In the following, a full characterization of the steadystate experiment has been performed, including computation of relative permeability (Sec. III A) and interfacial areas (Sec. III B), capillary pressure from interfacial curvature (Sec. III C), analysis of the shape of the fluid-fluid interface (Sec. III D), and wettability assessment by measuring in situ contact angles (Sec. III E).

\section{A. Saturation profile, pressure measurements, relative permeability, and pore filling}

At each fractional flow, the $1600 \times 1600 \times 3000$ voxel image was divided into 30 blocks along the flow direction. Each block contained $1600 \times 1600 \times 100$ voxels (approximately $0.36 \mathrm{~mm}$ in length along the sample). Figure 3 shows the averaged saturation profile in the field of view along the flow direction for each fractional flow at steady state. The total porosity used in this study to compute saturation, which was 0.231 , was obtained by the differential imaging method with high-salinity brine, step 13 in the protocol above $[20,28,37,40]$. It can be observed that in this system, which we will call mixed-wet, the saturation is relatively uniform along the flow direction. Within the imaged field of view, the capillary end effect, which can be indicated by a saturation gradient, was not observed. In a previous study with a water-wet sample, a clear saturation gradient was measured; this led to an analytical correction of the estimated relative permeabilities [20]. In this case we do not need to correct for the capillary end effect; as we show in Sec. 3.3, the capillary pressure is almost zero for most of the fractional flows. Note that the experiment spans a wide range of average brine saturation from approximately $10 \%$ initially to $90 \%$ at the end of the experiment.

Figure 4 shows the 3 -h pressure differential across the entire sample at each fractional flow before image acquisition started. The stable pressure differential indicates that the entire sample reached a steady-state condition. In a previous study on water-wet Bentheimer samples, we have observed larger pressure fluctuations which increased with the fractional flow reaching a maximum at $f_{w}=0.8$ [20,28,41], where the standard deviation in the measured pressure differential was almost $11 \mathrm{kPa}$. It was interpreted that these fluctuations represent local pore-scale rearrangements of the fluids with a magnitude consistent with the measured capillary pressure [20]. Here instead the largest standard deviation was only $0.12 \mathrm{kPa}$. This could represent simply instrument noise, or again represent pore-scale displacement, albeit at a much lower capillary pressure. As we will show in Sec. III C, the capillary pressures estimated from interfacial curvature are indeed this low.

With the computed saturation values and the pressure differentials, the relative permeability for both the oil and brine phases can be calculated [20]. The relative permeability for each phase at steady state is shown in Table II and plotted in Fig. 5.

The relative permeability for the oil phase at $f_{w}=0$ is higher than the corresponding value in the water-wet system; this is mainly due to the brine saturation at $f_{w}=0\left(S_{w}=0.115\right)$, which was initialized by high-speed centrifugation and hence is lower than in the water-wet case $\left(S_{w}=0.144\right)$ where the initial brine saturation was achieved by high flow rate flooding [20]. After reaching steady state at $f_{w}=1$, the relative permeability of the brine phase in the mixed-wet system $\left(k_{r o}=0.383\right)$ is much higher than in the water-wet case $\left(k_{r o}=0.079\right)$. In the mixed-wet rock, the remaining oil saturation $\left(S_{o}=1-S_{w}\right)$ is approximately 0.11 , which is substantially lower than in the water-wet case $\left(S_{o}=0.38\right)$. The crossover saturation point, where the relative permeability values for the oil and brine phases intersect, in the mixed-wet case is about 0.65 , whereas for the water-wet case, the crossover saturation point is lower, around 0.55 .

Our results are typical of a mixed-wet rock [32,42]: the drainage of oil layers allows a low oil saturation to be attained, even though the oil relative permeability is low at high brine saturation. The relative permeabilities cross at a high-brine saturation, indicating favorable waterflood recovery: this is because the brine relative permeability remains low until a high saturation is reached, since the brine stays poorly connected in the pore space. Using pore-scale imaging we can test this interpretation directly, as presented below.

First, we consider the filling sequence. To interpret our results, we performed a topological analysis of the pore space. We used a maximal ball method to locate pores, whose centers contain the largest spheres that can be fit into the void space. Pores are bounded by restrictions, called throats, which are the surfaces between two neighboring pores [43]. We define occupancy from the voxel nearest the pore center: this is filled with either oil or brine. Figure 6 shows a normalized histogram of the number of pores occupied by oil, weighted by the pore volume, as a function of pore radius (the 

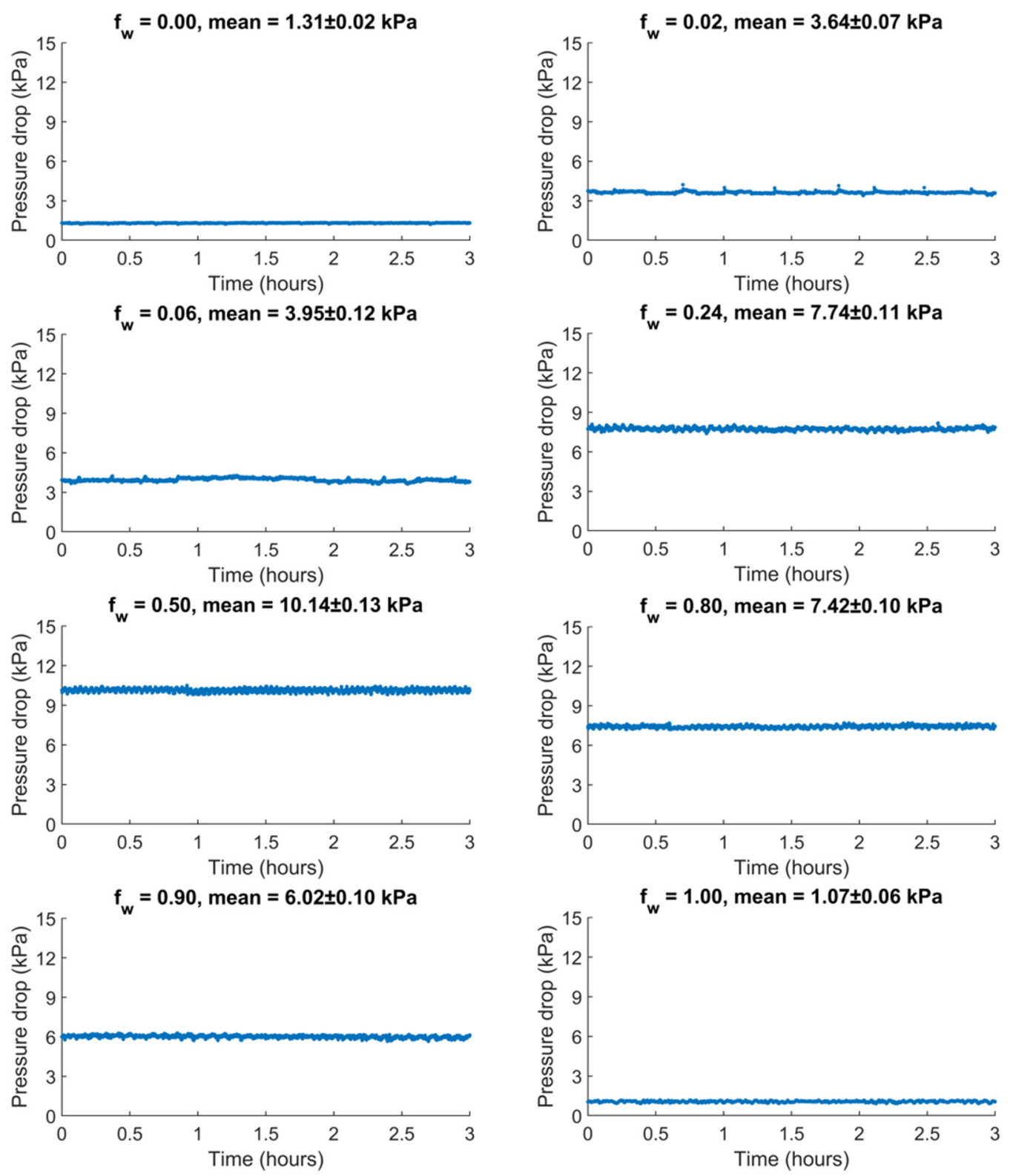

FIG. 4. The measured pressure differential across the sample using a differential pressure transducer at steady state for $3 \mathrm{~h}$ before starting the scan at different fractional flows. The mean pressure differential and standard deviation are shown.

radius of the maximal ball). All the histograms are normalized to the highest volume-weighted frequency of all of the pores.

For a water-wet case [20] oil initially resides in the larger pores while brine preferentially fills the smaller pores; during waterflooding brine occupies progressively wider regions while the oil ends up trapped in the bigger pores. In our mixed-wet experiment, where we assume that most interfaces become nonwetting to brine, brine now preferentially displaces oil from the larger pores, with oil trapped in narrower regions. While Fig. 6 does confirm this tendency, note that there is displacement in pores of all size with only a rather weak segregation in terms of pore radius. While almost all the very largest pores are eventually brine-filled, so too are many of the smaller elements.

\section{B. Interfacial areas}

In this section, the interfacial areas between different phases are presented. To compute the surface areas, the voxelized interface between two phases (fluid-fluid or fluidsolid) was extracted. Then a boundary-preserving surfacesmoothing algorithm was applied with 150 iterations [44].The surface-smoothing algorithm shifts the vertices of the original voxelized images such that each vertex is moved towards the average position of its neighbors. Figure 7 demonstrates the smoothed surface and its original voxelized interface. This operation removes the stair-step nature of the original interface.

Specific interfacial area, $a$, is defined as the surface area of an interface divided by the total volume considered: in this case the total volume of the entire scanned region. Figure 8 shows the specific interfacial area for interfaces between oil 


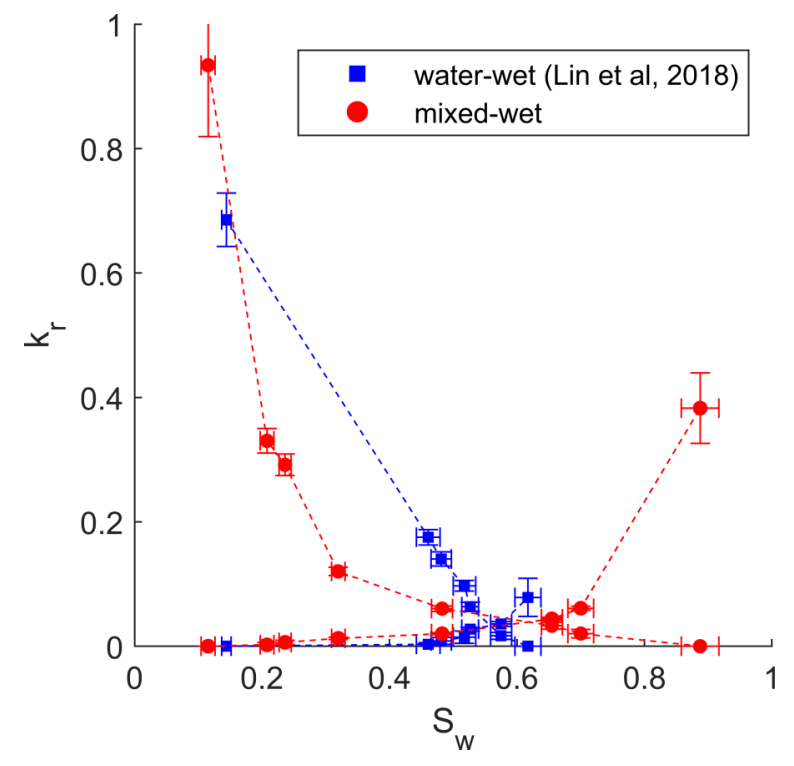

FIG. 5. Relative permeabilities during waterflood calculated at steady state for brine displacing oil for the mixed-wet Bentheimer system in this study are shown by the red circles. The result is compared to previously published measurements on a water-wet Bentheimer system performed at the same experimental conditions apart from sample wettability, shown by the blue squares [20]. The error bars consider uncertainties in the measurement of the length and diameter of the sample, the absolute permeability, pressure measurements, and the pump rates, as well as the sensitivity from image segmentation.

and solid phases $\left(a_{o s}\right)$, brine and solid phases $\left(a_{w s}\right)$, and oil and brine phases $\left(a_{o w}\right)$.

The interfacial areas were limited by the resolution of the image and did not capture fine-scale roughness on the surface or some oil-brine interfaces in the corners of the pore space. Overall, we see that the solid surface area is much larger than that between the fluids. As more brine invades the pore space, $a_{w s}$ increases while $a_{o w}$ decreases, as expected, with an approximately linear trend with saturation.

The oil-brine interfacial area is approximately constant over a wide range of saturation, dropping near both end points. These interfaces are principally terminal menisci [45] that block the pore space and prevent flow of either phase. The consequence of this is then evident in the relative permeabilities shown in Fig, 5: when $a_{o w}$ is large, the relative permeabilities, particularly of the brine phase, are low. Alhammadi et al.

TABLE II. Relative permeability of the oil and brine phases.

\begin{tabular}{llll}
\hline \hline $\boldsymbol{f}_{\boldsymbol{w}}$ & $\boldsymbol{S}_{\boldsymbol{w}}$ & $\boldsymbol{k}_{\boldsymbol{r} \boldsymbol{o}}$ & $\boldsymbol{k}_{\boldsymbol{r} \boldsymbol{w}}$ \\
\hline 0 & 0.115 & 0.934 & 0 \\
0.02 & 0.208 & 0.33 & 0.002 \\
0.06 & 0.236 & 0.292 & 0.006 \\
0.24 & 0.32 & 0.12 & 0.013 \\
0.5 & 0.482 & 0.06 & 0.02 \\
0.8 & 0.655 & 0.033 & 0.044 \\
0.9 & 0.7 & 0.02 & 0.061 \\
1 & 0.887 & 0 & 0.383 \\
\hline \hline
\end{tabular}
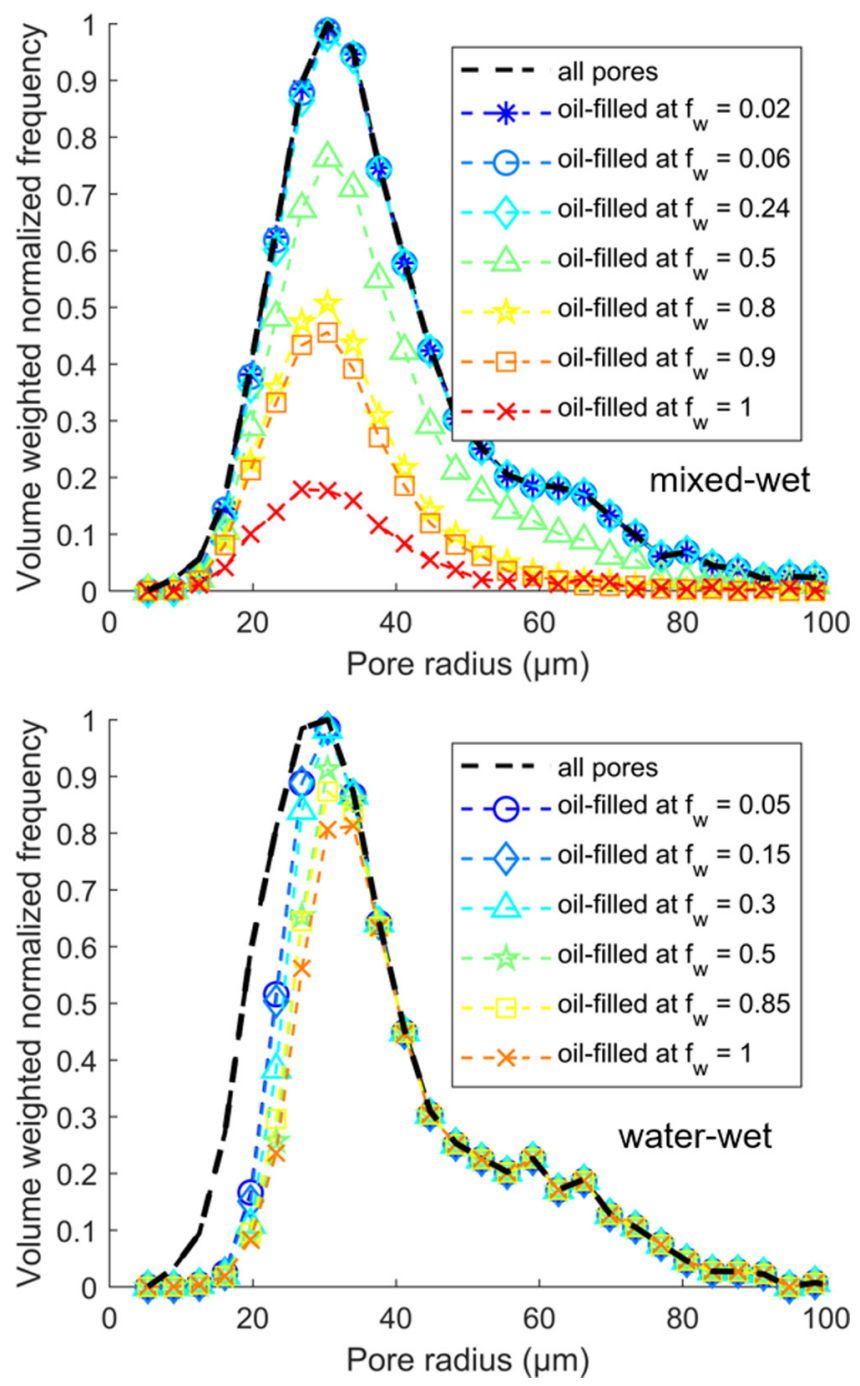

FIG. 6. Normalized histograms showing the number of pores occupied by oil, weighted by the pore volume, as a function of pore radius for different fractional flows. The mixed-wet case (top) is compared with a previous water-wet experiment (bottom) [20].

[9] computed the specific interfacial area $a_{o w}$ at remaining oil saturations for one weakly water-wet and two mixed-wet (with different levels of wettability alteration) carbonate reservoir samples. The specific oil-brine interfacial areas for the two mixed-wet carbonate cases were $8.52 \times 10^{-3} \mu \mathrm{m}^{-3}$ $\left(S_{w}=0.59\right.$, a more oil-wet case $)$ and $4.26 \times 10^{-3} \mu \mathrm{m}^{-3}$ $\left(S_{w}=0.84\right)$, which was higher than $3.02 \times 10^{-3} \mu \mathrm{m}^{-3}$ $\left(S_{w}=0.67\right)$ for the weakly water-wet sample. For the mixedwet Bentheimer sandstone in this study, at the remaining oil saturation $\left(S_{w}=0.89\right)$, the specific interfacial area is $3.58 \times$ $10^{-3} \mu \mathrm{m}^{-3}$, which is comparable with these previous results, indicating weakly water-wet to mixed-wet behavior. Culligan et al. $[14,46]$ also used x-ray microtomography to measure interfacial area in a water-wet bead pack. Their specific areas were smaller than ours, simply due to the larger pore size, and showed a sharp increase at low brine saturation reaching a maximum at around 0.3 due to the swelling of wetting layers; here in a mixed-wet rock, brine is no longer the wetting phase, and the maximum is pushed to higher saturation. 

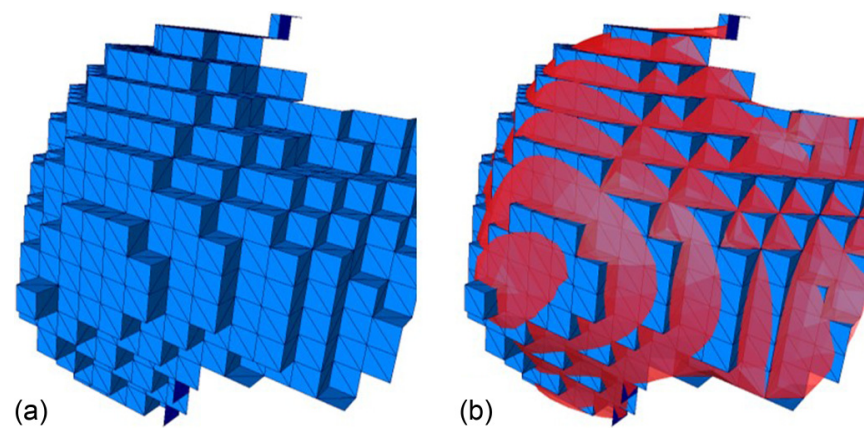

FIG. 7. (a) The voxelized surface interfaces between two phases. (b) The voxelized interfaces were smoothed by applying a boundarypreserving algorithm with 150 iterations, shown in red overlaid on top of the voxelized interface (in blue).

\section{Interfacial curvature and capillary pressure}

The capillary pressure, which can be defined as the pressure difference between the oil and brine, can be obtained by measuring the interfacial curvature and then applying the Young-Laplace law [16,20]:

$$
\begin{gathered}
P_{c}=P_{o}-P_{w}, \\
P_{c}=2 \sigma \kappa, \\
\kappa=\frac{1}{2}\left(\kappa_{1}+\kappa_{2}\right),
\end{gathered}
$$

where $\sigma$ is the interfacial tension between the brine and oil phases, and $\kappa$ is the mean curvature of the interface, which is the average of the two principal curvatures $\left(\kappa_{1}\right.$ and $\kappa_{2}$

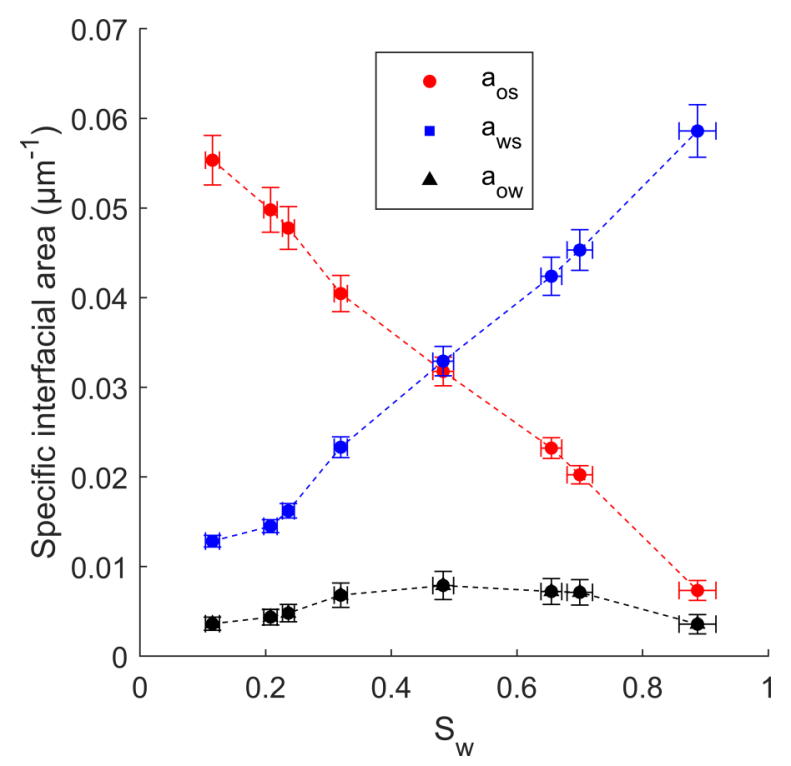

FIG. 8. Specific interfacial area, $a$, between different phases calculated at steady state for brine-oil displacement. The specific surface is defined as the surface area per unit volume. $a_{o s}, a_{w s}$, and $a_{o w}$ represent specific interfacial area between oil and solid phases (red circle), brine and solid phases (blue square), and oil and brine phases (black triangle), respectively. The error bars consider the sensitivity from image segmentation.

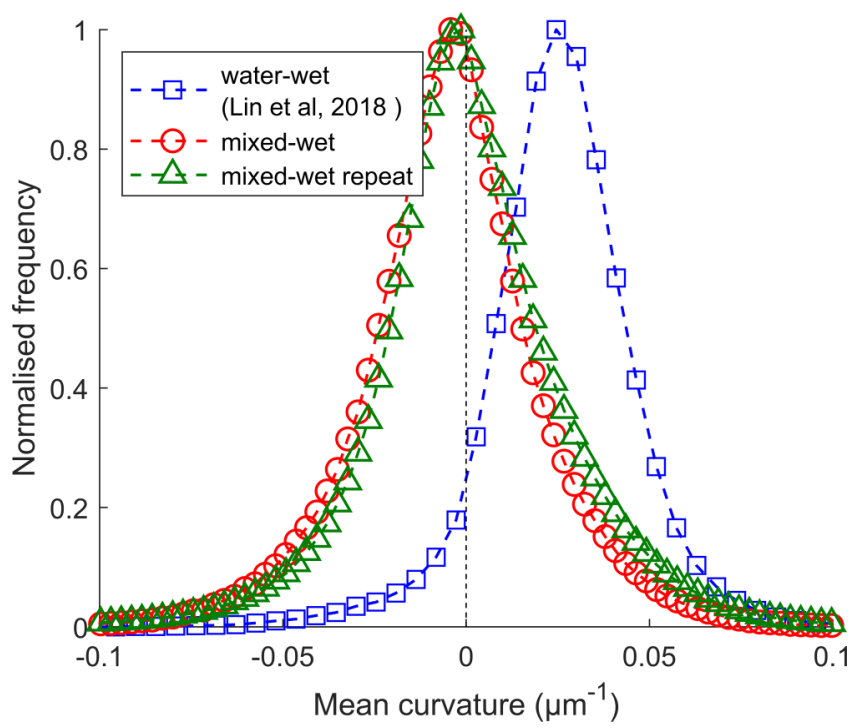

FIG. 9. Curvature distributions for the two mixed-wet experiments considered and a water wet case from a previous study [20]. All the curvature distributions were computed with $f_{w}=0.5$ at steady state.

where we define $\kappa_{1}>\kappa_{2}$ ). The curvature was found in our experiments by approximating the smoothed oil-brine surface locally as a quadratic form. The eigenvalues and eigenvectors of this quadratic form represent the principal curvature values and the directions of principal curvature, respectively. A surface scalar field was produced where the principal radii of curvature were averaged and assigned to each element across the interface $[16,20]$. In the curvature measurement, to reduce uncertainty caused by segmentation at the three-phase contact line [19], values close to the rock surface (less than 3 voxels away) were not included in the analysis. The mean curvature distributions for the main and repeat experiment and a water-wet case at $f_{w}=0.5$ are compared in Fig. 9. We see that the average curvature is close to zero and reproducible between the two mixed-wet experiments.

The mean curvatures for different fractional flows for each block of $1600 \times 1600 \times 100$ voxels (30 blocks for the entire volume) are shown in Fig. 10. We considered three cases: first we analyzed only interfaces where the brine was in contact with oil that connected across the entire volume of $1600 \times$ $1600 \times 3000$ voxels (see Fig. 3), second only the interfaces between brine and disconnected oil were looked at, and finally all interfaces were considered.

With Eq. (2), the capillary pressure can be obtained. The $P_{c}-S_{w}$ relationship for all fractional flows is shown in Fig. 11 . The capillary pressure in this mixed-wet system is compared with a centrifuge measurement on a larger sample and is also compared with a previous study on water-wet Bentheimer sandstone [20]. The mean capillary pressures at different fractional flows with standard deviation values are shown in Table III.

The first observation is that the magnitude of the mean curvature and capillary pressure are approximately 10 times lower in the mixed-wet case, compared to the water-wet measurements; see Figs. 9, 10, and 11. The mean curvature 

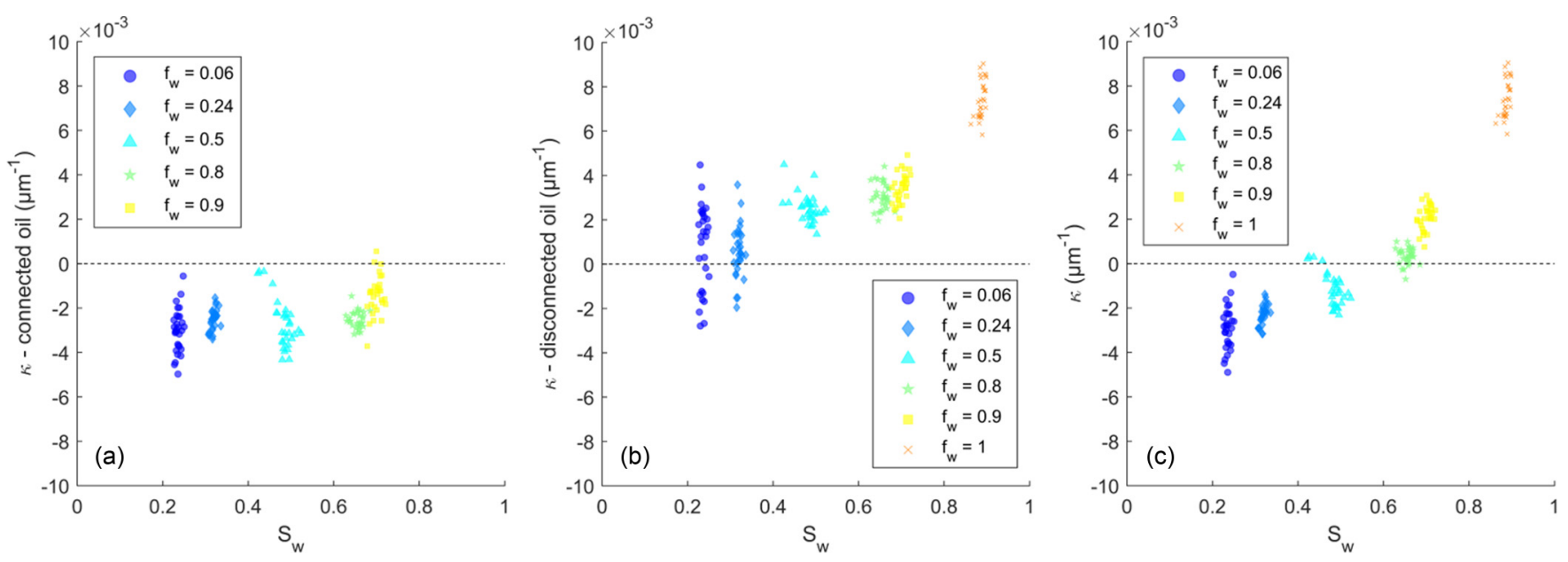

FIG. 10. The mean curvatures $(\kappa)$ for different fractional flows for all the subvolume blocks. Mean curvatures are measured using the connected oil phase only (a), disconnected oil phase only (b), and all oil-brine interfaces (c). Note that there is no connected oil phase when $f_{w}=1$ at steady state.

is close to zero (Figs. 9 and 10), which indicates an overall rather weakly oil-wet rock. This will be tested directly when contact angles are measured in Sec. III E.

The capillary pressure remains approximately constant over a wide saturation range (Fig. 11). After primary drainage, we can assume that all the surfaces contacted by oil alter their wettability such that a negative capillary pressure is required for displacement: brine has to push oil out of the pore space. The brine will preferentially fill the larger pores if it is the nonwetting phase. However, the displacement has to be initiated from initial brine that is confined to the narrower regions of the pore space. Hence the brine has to force its way out of these restrictions to fill a pore (this is a forced snap-off process $[32,48]$ ), which occurs at a negative capillary pressure. Once this threshold is overcome, it is possible for brine then to displace a significant fraction of the oil at more or less the same capillary pressure; furthermore, the oil-brine interfacial area (see Fig. 8) is also almost constant, implying that menisci simply move through the pore space, rather than create new interfaces as more oil is removed. This is also supported by the rather weak preference for filling on the basis of size (Fig. 6): menisci are able to progress through the void space with little impediment based on local pore radius. Also note that the brine phase has a low relative permeability (see Fig. 5) until a high brine saturation is reached, since the brine can flow readily only once there is a connected pathway of larger brine-filled pores across the rock.

When trapped oil is considered, the capillary pressure apparently increases with saturation [17]. It has been observed in synchrotron experiments with fast imaging that when oil becomes disconnected it rearranges in the pore space to have a higher overall pressure: this is seen in both drainage and water injection $[3,49]$. The rearrangement in this case leads to a higher local capillary pressure than the prevailing value in the connected phases. The capillary pressure to use in larger-scale modeling is, however, the pressure difference, between the connected phases: this is, to within the scatter of the measurements, constant over an intermediate saturation range.
The observation of a small capillary pressure could imply that the oil-brine interfaces are largely flat; however, as we show in the next section, this is not the case, as the interfaces typically have two equal but opposite curvatures.

\section{Shape of the interface and Gaussian curvature}

Figure 12 shows the interfaces between connected oil and brine in the water-wet case from the previous study and two mixed-wet experiments. The images shown here are at $f_{w}=0.5$ with a dimension of $250 \times 250 \times 250$ voxels (approximately $0.73 \mathrm{~mm}^{3}$ ). The brine saturations for the water-wet case, mixed-wet case, and repeated mixed-wet case are $0.527,0.482$, and 0.502 , respectively. Qualitatively, we see that for the water-wet rock, the interfaces are quasispherical, surrounding oil in the larger pore spaces. The mixed-wet cases are similar to each other but display a more complex arrangement of the interfaces which have a wavier character. To describe these complex interfaces in the mixed-wet system with mean curvatures close to zero, we introduce Gaussian curvature $\left(K=\kappa_{1} \kappa_{2}\right)$ [50,51].

For a water-wet rock, where the oil resides as quasispherical blobs in the centers of the largest pores, the curvature along the two principal axes are similar and positive, i.e., biconvex, $\kappa_{1}>0, \kappa_{2}>0$. Hence, both the mean curvature $\kappa$ and the Gaussian curvature are positive. In the opposite case, a strongly oil-wet rock, we might anticipate that here water resides as spherical droplets with an interface that bulges into the oil phase, i.e., biconcave. In this case, again, the curvatures are similar but negative, and hence we would see large and negative values of $\kappa$ but a large positive Gaussian curvature. However, in a mixed-wet case, we see interfaces with significant curvature of a similar magnitude to that observed in a water-wet rock; however, the curvature in one direction $\left(\kappa_{1}\right)$ is almost equal in magnitude and opposite in sign to the curvature in a perpendicular direction $\left(\kappa_{2}\right)$, which causes the mean curvature to be small and close to zero. Oil can bulge into brine one way, while brine can bulge into oil in the other, forming complex interfaces. Thus, $\kappa_{1} \kappa_{2} \leqslant 0$. 

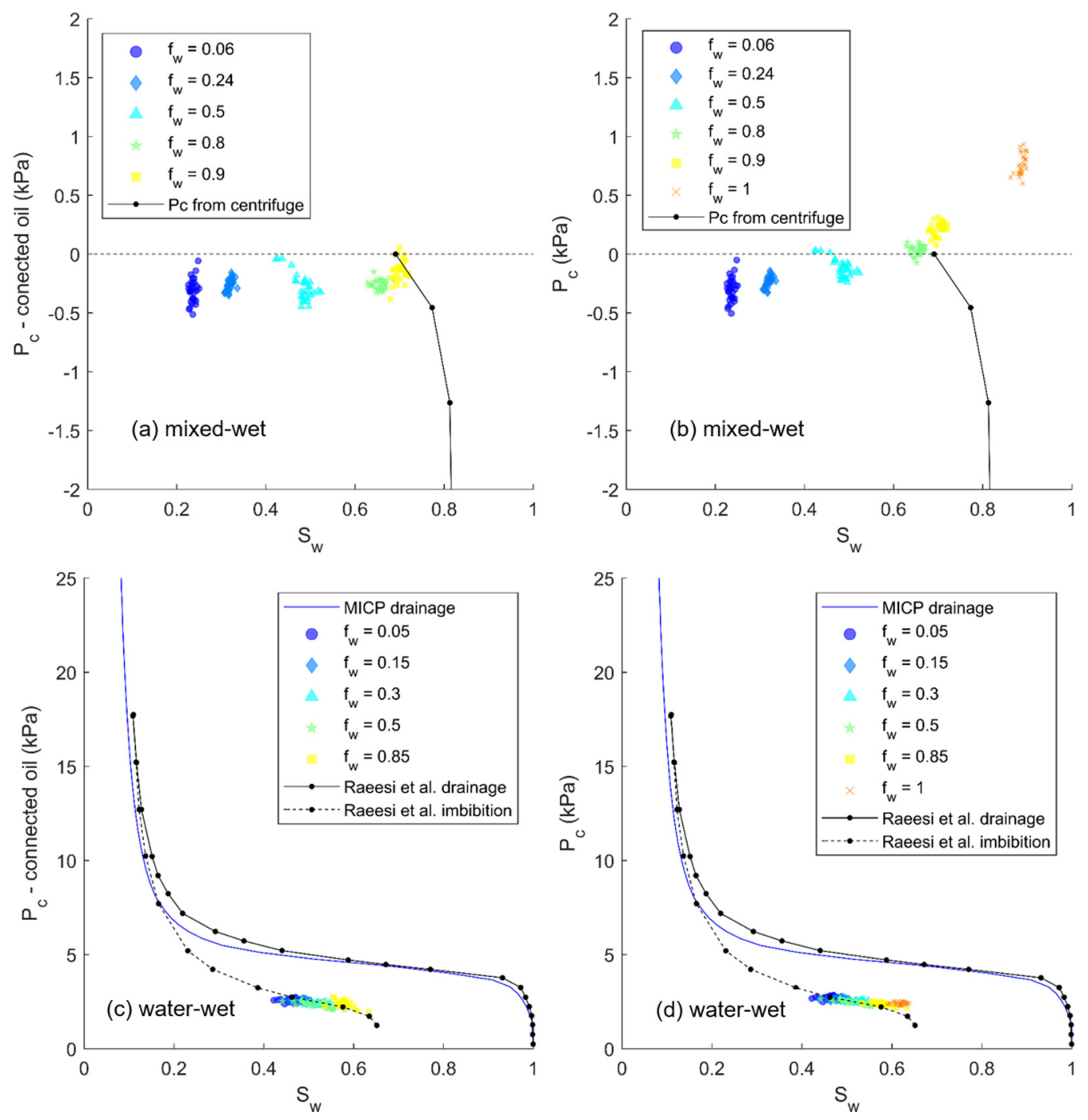

FIG. 11. The average capillary pressure for (a) the connected oil phase only and (b) all of the oil phase against average saturation for each block along the flow direction at different fractional flows. The capillary pressure obtained using a centrifuge method on a larger system is also compared to the measurement based on interfacial curvature. The capillary pressure in this mixed-wet system is also compared with the previous study on a water-wet sample (c, d) [20,47]. Note that in both mixed-wet and water-wet cases, there is no connected oil phase when $f_{w}=1$ at steady state.

To quantify this observation for all the interfaces, the mean curvature distribution for the mixed-wet experiment was divided into three categories, $\kappa_{1}<0, \kappa_{2}<0$; $\kappa_{1}>0, \kappa_{2}>0$; and $\kappa_{1} \kappa_{2} \leqslant 0$, shown in Fig. 13 .

Figure 14 shows the Gaussian curvature distribution for the water-wet and mixed-wet cases. We see that indeed in the water-wet case most interfaces have two positive curvatures and consequently a positive Gaussian curvature. For the mixed-wet case, the opposite is observed where most of the Gaussian curvatures are negative.

This result is more than a geometrical curiosity, but represents the topological origin of the favorable oil recoveries indicated by the relative permeabilities and low residual oil saturation (Fig. 5): a negative Gaussian curvature implies a well-connected object [52]. In this case, the oil is well connected in the pore space allowing it to flow to low saturation in layers. In contrast, in a more strongly wetted medium, with a positive Gaussian curvature, the oil phase can be trapped or retained in the pore space with a poorer connectivity and lower recovery. Hence this anal-

TABLE III. The mean capillary pressure at different fraction flows with the standard deviation from measurements in different subvolume blocks. The left-hand columns consider connected oil only, while the right-hand columns consider all interfaces.

\begin{tabular}{llllll}
\hline \hline & \multirow{2}{*}{$P_{c}$-connected oil $(\mathrm{kPa})$} & & \multicolumn{2}{c}{$P_{c}(\mathrm{kPa})$} \\
\cline { 2 - 3 } \cline { 5 - 6 }$f_{w}$ & Mean & Std & & Mean & Std \\
\hline 0.06 & -0.311 & 0.101 & & -0.301 & 0.101 \\
0.24 & -0.257 & 0.048 & & -0.235 & 0.046 \\
0.5 & -0.28 & 0.114 & & -0.12 & 0.078 \\
0.8 & -0.26 & 0.039 & & 0.035 & 0.039 \\
0.9 & -0.146 & 0.092 & & 0.215 & 0.059 \\
1 & N/A & N/A & 0.765 & 0.088 \\
\hline \hline
\end{tabular}




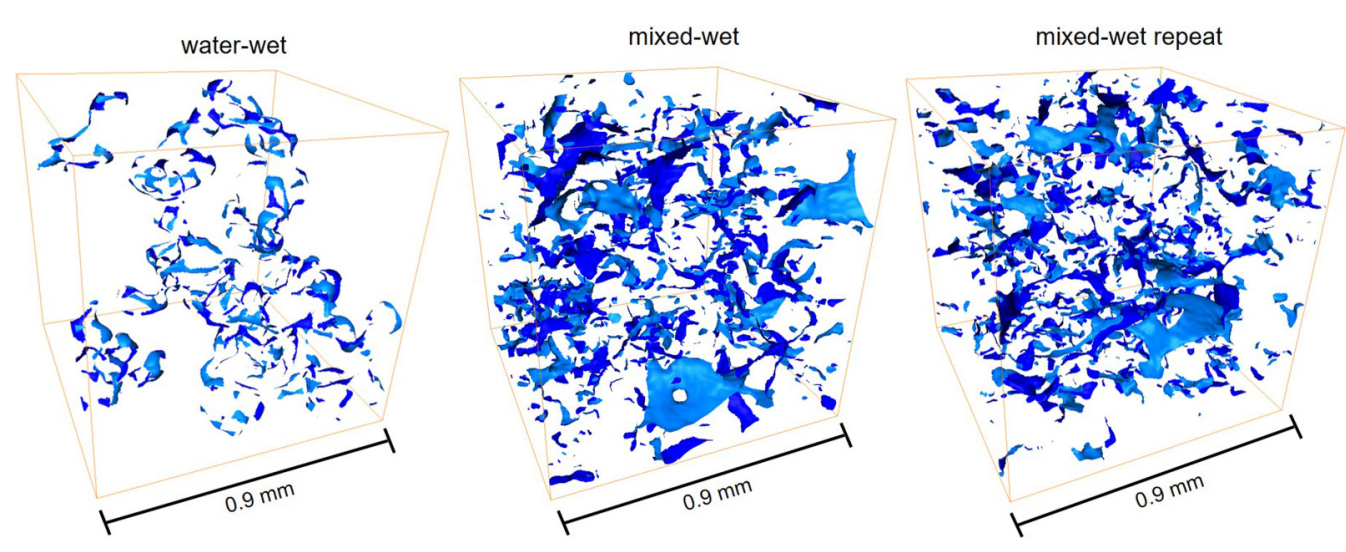

FIG. 12. Interfaces between connected oil phase and brine phase in the water-wet case from a previous study [20] and two mixed-wet experiments. The images shown here are at $f_{w}=0.5$ with a dimension of $250 \times 250 \times 250$ voxels (approximately $0.73 \mathrm{~mm}^{3}$ )

ysis provides a fundamental observation concerning some altered wettability rocks, namely, that the saddle shape of the fluid-fluid interface implies good connectivity of the phases.

The fluid-fluid interface is approximately a minimal surface $\left(\kappa_{1}=-\kappa_{2}\right)$, which is observed in many other systems where two phases preserve good connectivity, including emulsions [53], soap films and foams [54], spongy carbon [55], and peptide structures in cells [56]. In the context of porous media, we have a complex void space geometry with surfaces that are nonwetting to water. What is, theoretically, the most efficient manner to displace oil? This is when the capillary pressure is as close to zero as possible (it cannot be positive if the surfaces are oil-wet) and where the interfacial area is minimized. This is exactly what we see here: almost zeromean curvature interfaces that are locally saddle-shaped and that move through the rock displacing the vast majority of the oil with little change in either interfacial area or capillary pressure. However, we also see a wide distribution of curvature, which appears inconsistent with capillary equilibrium. It is likely that some of this scatter is due to imaging artifacts and the difficulty associated with capturing curvature accurately from voxelized images. Further work with perhaps higherresolution images is required before the concept of minimal surfaces in porous media can be developed further.

\section{E. In situ contact angle measurements}

Measuring an in situ contact is an alternative way to characterize the wettability of the system. Recently, an automated contact angle measurement algorithm has been developed and has been applied to measure wettability in different rock samples [9,10].

In this study, the automated algorithm was applied to compute the contact angle distribution for the mixed-wet system and to compare it to the water-wet system studied previously [20]; the results are shown in Fig. 15. Due to the large image size, the contact angles were computed from a subvolume with $500 \times 500 \times 500$ voxels from the center of the entire image for each fractional flow.
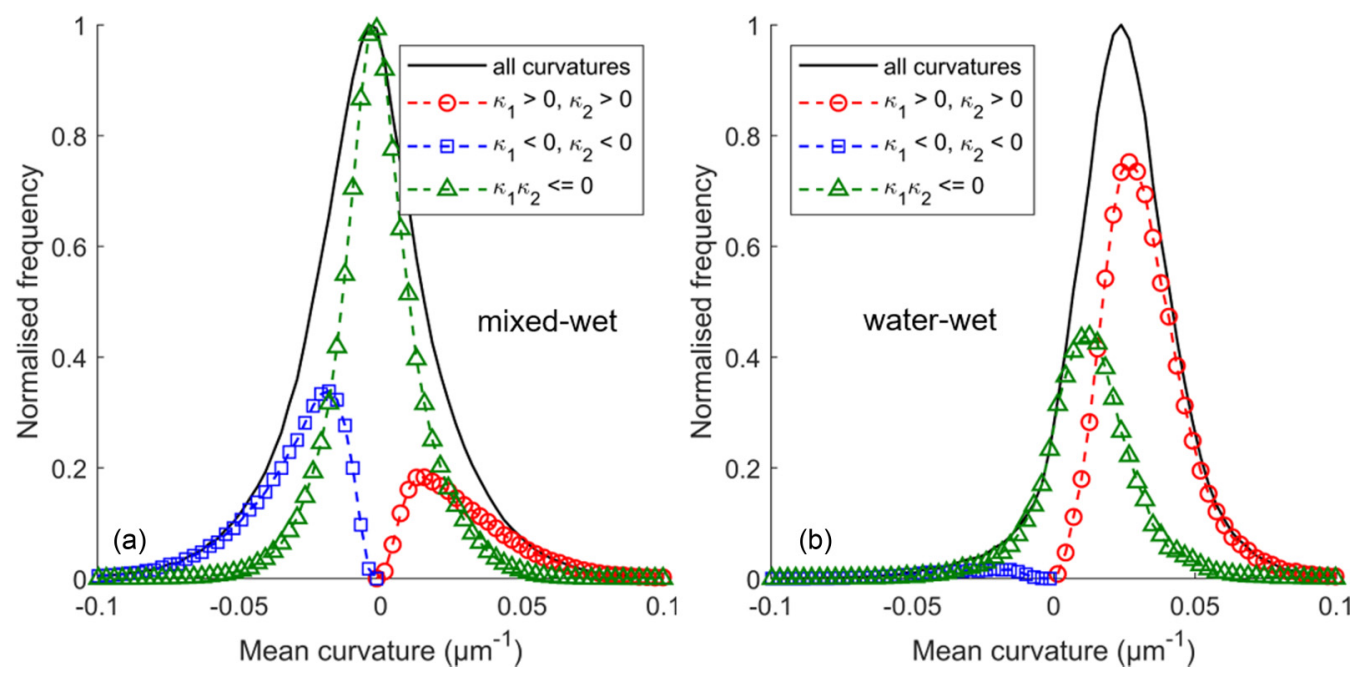

FIG. 13. Distributions showing different curvature values for $\kappa_{1}<0, \kappa_{2}<0$ (blue squares); $\kappa_{1}>0, \kappa_{2}>0$ (red circles), and $\kappa_{1} \kappa_{2} \leqslant 0$ (green triangles). Approximately $70 \%$ of the interfaces in the mixed-wet system have a negative Gaussian curvature. This phenomenon occurs at all fractional flows. The same plot for the water-wet case is shown here for comparison where the majority of the interfaces have a positive Gaussian curvature $\kappa_{1} \kappa_{2}>0\left(\kappa_{1}>0\right.$ and $\left.\kappa_{2}>0\right)$. 


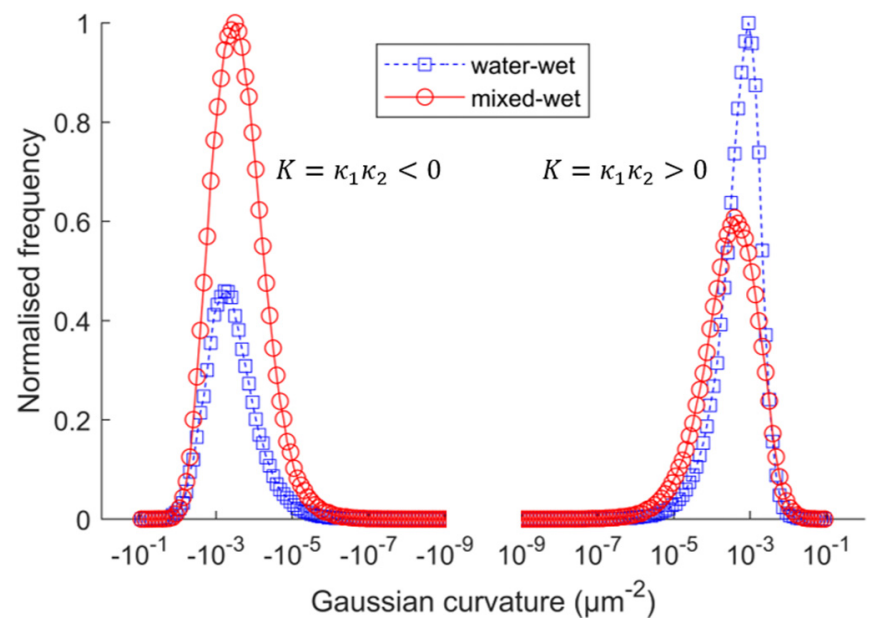

FIG. 14. Distribution of Gaussian curvature for the water-wet (blue squares) and mixed-wet (red circles) cases.

The contact angle measurement was made at steady state and was calculated at the three-phase contact line where image segmentation is most challenging. These values therefore have some uncertainty and do not necessarily indicate the advancing angles needed for displacement: due to contact angle hysteresis these values are likely to be larger [45,57].

There is a clear difference in the contact angle distribution for the two cases: while the shape and standard deviations are similar, the mean value is $14^{\circ}$ higher for the mixed-wet sample. Note that even after contact with crude oil, the apparent distribution of contact angle indicates rather neutrally wet or even weakly water-wet conditions. This is consistent with the very low capillary pressure, where values close to 0 are indicative of contact angles around $90^{\circ}$ [32]. However, this measurement does not directly indicate the most surprising

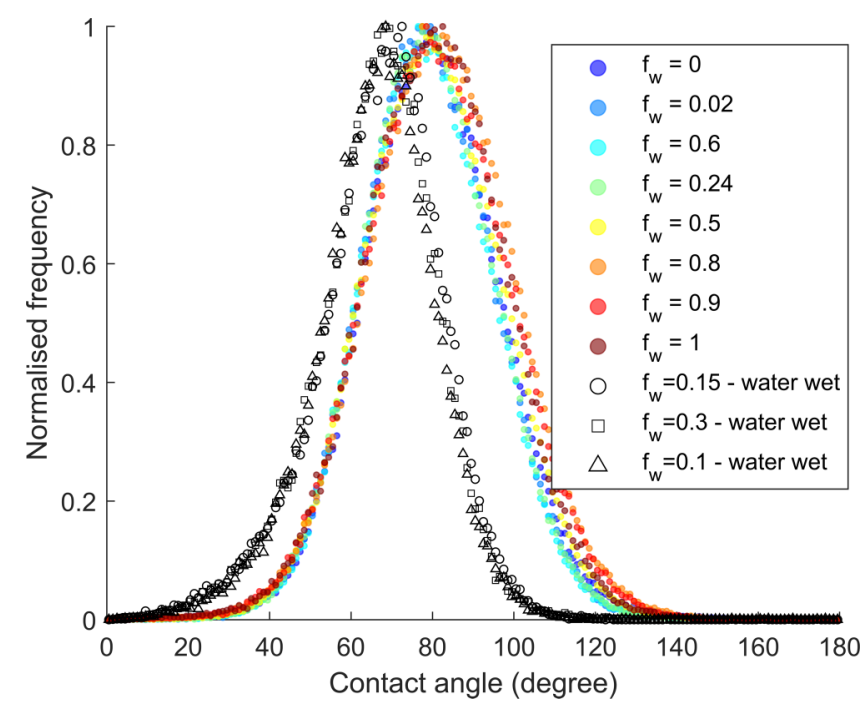

FIG. 15. Contact angle distribution computed from a $500 \times$ $500 \times 500$ voxels subvolume at each fractional flow. The mean contact angle for the mixed-wet experiment is $80.3^{\circ}$ with a standard deviation of $17.0^{\circ}$. The contact angle distribution is also compared with the water wet system [20], which has a mean contact angle of $66.4^{\circ}$ with a standard deviation of $15.1^{\circ}$. finding of this study: the presence of interfaces with significant positive and negative curvature in orthogonal directions. The fact that we do not observe contact angles significantly above $90^{\circ}$, indicating more strongly oil-wet conditions, is because we find effective values at rest on rough surfaces. Here the brine retained in the corners of the pore space renders the solid, on average, less oil-wet than a smooth surface of the same mineralogy $[9,58]$.

\section{CONCLUSIONS AND OUTLOOK}

We have demonstrated a methodology to measure relative permeability and capillary pressure simultaneously from a steady-state water-flood experiment on a small rock sample whose wettability had been altered through contact with crude oil. Pore-scale imaging provides an accurate measurement of saturation, while allowing interfacial area, curvature, and contact angle to be analyzed.

The sample displayed mixed-wet behavior, with a small negative capillary pressure whose magnitude is one tenth that measured on a similar water-wet rock. The capillary pressure and fluid-fluid interfacial area were approximately constant over a wide range of saturation. The measured contact angles at rest averaged $80^{\circ}$ with a standard deviation of $17^{\circ}$; with hysteresis the contact angles for displacement are likely to be higher. There was only a weak tendency for the water to favor filling in larger pores.

The fluid-fluid interfaces were not flat but had curvatures of approximately equal magnitude but opposite sign in orthogonal directions. These are minimal surfaces. The relative permeabilities imply favorable waterflood recovery with a low residual saturation of 0.11 , consistent with larger-scale measurements. Zero-mean curvature interfaces passed through the pore space displacing oil with little change in capillary pressure or interfacial area.

We suggest that the same approach could be used more widely to characterize the flow properties of rock samples, for the design of improved oil recovery, as well as for the management of carbon dioxide storage and groundwater pollution. The combination of pore-scale imaging with macroscopic measurements provides a major improvement in our ability to understand multiphase flow processes. The images provide a careful quality control of the results while allowing a complete characterization of interfacial areas, curvature, and wettability, as well as providing benchmark data sets to validate pore-scale models.

Current pore-scale models and theoretical analysis generally do not even entertain the possibility of saddle-shaped menisci spanning the pore space, with curvatures of opposite sign in orthogonal directions. We have shown that this condition may in fact be typical of multiphase flow in natural systems.

\section{ACKNOWLEDGMENTS}

We gratefully acknowledge funding from the Shell Digital Rocks programme at Imperial College London. We thank O. Wilson, Ab. Coorn, and H. van der Linde from Shell for sample preparation, as well as helpful and insightful comments on this work. 
[1] M. J. Blunt, B. Bijeljic, H. Dong, O. Gharbi, S. Iglauer, P. Mostaghimi, A. Paluszny, and C. Pentland, Pore-scale imaging and modelling, Adv. Water Resour. 51, 197 (2013).

[2] D. Wildenschild and A. P. Sheppard, X-ray imaging and analysis techniques for quantifying pore-scale structure and processes in subsurface porous medium systems, Adv. Water Resour. 51, 217 (2013).

[3] M. Andrew, H. Menke, M. J. Blunt, and B. Bijeljic, The imaging of dynamic multiphase fluid flow using synchrotron-based $\mathrm{x}$-ray microtomography at reservoir conditions, Transp. Porous Media 110, 1 (2015).

[4] S. Berg, M. Rücker, H. Ott, A. Georgiadis, H. van der Linde, F. Enzmann, M. Kersten, R. T. Armstrong, S. de With, J. Becker, and A. Wiegmann, Connected pathway relative permeability from pore-scale imaging of imbibition, Adv. Water Resour. 90, 24 (2016).

[5] S. Berg, H. Ott, S. A. Klapp, A. Schwing, R. Neiteler, N. Brussee, A. Makurat, L. Leu, F. Enzmann, J.-O. Schwarz, M. Kersten, S. Irvine, and M. Stampanoni, Real-time 3D imaging of Haines jumps in porous media flow, Proc. Natl. Acad. Sci. USA 110, 3755 (2013).

[6] T. Pak, I. B. Butler, S. Geiger, M. I. J. van Dijke, and K. S. Sorbie, Droplet fragmentation: 3D imaging of a previously unidentified pore-scale process during multiphase flow in porous media, Proc. Natl. Acad. Sci. USA 112, 1947 (2015).

[7] K. Singh, H. Menke, M. Andrew, Q. Lin, C. Rau, M. J. Blunt, and B. Bijeljic, Dynamics of snap-off and pore-filling events during two-phase fluid flow in permeable media, Sci. Rep. 7, 5192 (2017).

[8] C. A. Reynolds, H. Menke, M. Andrew, M. J. Blunt, and S. Krevor, Dynamic fluid connectivity during steady-state multiphase flow in a sandstone, Proc. Natl. Acad. Sci. USA 114, 8187 (2017).

[9] A. M. Alhammadi, A. AlRatrout, K. Singh, B. Bijeljic, and M. J. Blunt, In situ characterization of mixed-wettability in a reservoir rock at subsurface conditions, Sci. Rep. 7, 10753 (2017).

[10] A. AlRatrout, A. Q. Raeini, B. Bijeljic, and M. J. Blunt, Automatic measurement of contact angle in pore-space images, Adv. Water Resour. 109, 158 (2017).

[11] M. Andrew, B. Bijeljic, and M. J. Blunt, Pore-scale contact angle measurements at reservoir conditions using X-ray microtomography, Adv. Water Resour. 68, 24 (2014).

[12] M. Khishvand, A. H. Alizadeh, and M. Piri, In-situ characterization of wettability and pore-scale displacements during two- and three-phase flow in natural porous media, Adv. Water Resour. 97, 279 (2016).

[13] M. Prodanovic, W. B. Lindquist, and R. S. Seright, 3D microtomographic study of fluid displacement in rock cores, in Proceedings, Computational Methods in Water Resources XV Conference, Chapel Hill, NC, June 13-17, 2004 (2004), pp. 223-234.

[14] K. A. Culligan, D. Wildenschild, B. S. B. Christensen, W. G. Gray, M. L. Rivers, and A. F. B. Tompson, Interfacial area measurements for unsaturated flow through a porous medium, Water Resour. Res. 40, W12413 (2004).

[15] M. Andrew, B. Bijeljic, and M. J. Blunt, Pore-by-pore capillary pressure measurements using X-ray microtomography at reservoir conditions: Curvature, snap-off, and remobilization of residual $\mathrm{CO}_{2}$, Water Resour. Res. 50, 8760 (2014).
[16] R. T. Armstrong, C. H. Pentland, S. Berg, J. Hummel, D. Lichau, and L. Bernard, Estimation of curvature from micro-CT liquid-liquid displacement studies with pore scale resolution, in Proceedings of the International Symposium of the Society of Core Analysts, Aberdeen, Scotland, UK, 27-30 August 2012 (Aberdeen, Scotland, UK, 2012), Vol. SCA2012-55.

[17] R. T. Armstrong, M. L. Porter, and D. Wildenschild, Linking pore-scale interfacial curvature to column-scale capillary pressure, Adv. Water Resour. 46, 55 (2012).

[18] C. Garing, J. A. de Chalendar, M. Voltolini, J. B. Ajo-Franklin, and S. M. Benson, Pore-scale capillary pressure analysis using multi-scale X-ray micromotography, Adv. Water Resour. 104, 223 (2017).

[19] T. Li, S. Schlüter, M. I. Dragila, and D. Wildenschild, An improved method for estimating capillary pressure from 3D microtomography images and its application to the study of disconnected nonwetting phase, Adv. Water Resour. 114, 249 (2018).

[20] Q. Lin, B. Bijeljic, R. Pini, M. J. Blunt, and S. Krevor, Imaging and measurement of pore-scale interfacial curvature to determine capillary pressure simultaneously with relative permeability, Water Resour. Res. 54, 7046 (2018).

[21] G. Hirasaki, Wettability: Fundamentals and surface forces, SPE Form. Eval. 6, 217 (1991).

[22] K. Nordahl and P. S. Ringrose, Identifying the representative elementary volume for permeability in heterolithic deposits using numerical rock models, Math. Geosci. 40, 753 (2008).

[23] S. Berg, S. Oedai, and H. Ott, Displacement and mass transfer between saturated and unsaturated $\mathrm{CO}_{2}$-brine systems in sandstone, Int. J. Greenhouse Gas Control 12, 478 (2013).

[24] S. J. Jackson, S. Agada, C. A. Reynolds, and S. Krevor, Characterizing drainage multiphase flow in heterogeneous sandstones, Water Resour. Res. 54, 3139 (2018).

[25] C. A. Reynolds, M. J. Blunt, and S. Krevor, Multiphase flow characteristics of heterogeneous rocks from $\mathrm{CO}_{2}$ storage reservoirs in the United Kingdom, Water Resour. Res. 54, 729 (2018).

[26] R. Pini, S. C. M. Krevor, and S. M. Benson, Capillary pressure and heterogeneity for the $\mathrm{CO}_{2}$ /water system in sandstone rocks at reservoir conditions, Adv. Water Resour. 38, 48 (2012).

[27] T. Bultreys, Q. Lin, Y. Gao, A. Q. Raeini, A. AlRatrout, B. Bijeljic, and M. J. Blunt, Validation of model predictions of pore-scale fluid distributions during two-phase flow, Phys. Rev. E 97, 053104 (2018).

[28] Y. Gao, Q. Lin, B. Bijeljic, and M. J. Blunt, X-ray microtomography of intermittency in multiphase flow at steady state using a differential imaging method, Water Resour. Res. 53, 10274 (2017).

[29] S. Zou, R. T. Armstrong, J.-Y. Arns, C. H. Arns, and F. Hussain, Experimental and theoretical evidence for increased ganglion dynamics during fractional flow in mixed-wet porous media, Water Resour. Res. 54, 3277 (2018).

[30] R. Lenormand, C. Zarcone, and A. Sarr, Mechanisms of the displacement of one fluid by another in a network of capillary ducts, J. Fluid Mech. 135, 337 (1983).

[31] H. S. Rabbani, B. Zhao, R. Juanes, and N. Shokri, Pore geometry control of apparent wetting in porous media, Sci. Rep. 8, 15729 (2018). 
[32] M. J. Blunt, Multiphase Flow in Permeable Media: A PoreScale Perspective (Cambridge University Press, Cambridge, 2017).

[33] J. Pérez, A new golden age of minimal surfaces, Notices Am, Math. Soc. 64, 347 (2017).

[34] Q. Lin, B. Bijeljic, S. C. Krevor, M. J. Blunt, M. Rücker, S. Berg, A. Coorn, H. van der Linde, A. Georgiadis, and O. B. Wilson, A new waterflood initialization protocol with wettability alteration for pore-scale multiphase flow experiments, Petrophysics 60, 264 (2019).

[35] J. M. Andreas, E. A. Hauser, and W. B. Tucker, Boundary tension by pendant drops 1 , J. Phys. Chem. 42, 1001 (1937).

[36] C. E. Stauffer, The measurement of surface tension by the pendant drop technique, J. Phys. Chem. 69, 1933 (1965).

[37] Q. Lin, Y. Al-Khulaifi, M. Blunt, and B. Bijeljic, Quantification of sub-resolution porosity in carbonate rocks by applying highsalinity contrast brine using X-ray microtomography differential imaging, Adv. Water Resour. 96, 306 (2016).

[38] A. Buades, B. Coll, and J.-M. Morel, Nonlocal image and movie denoising, Intl. J. Comput. Vision 76, 123 (2008).

[39] A. C. Jones, C. H. Arns, A. P. Sheppard, D. W. Hutmacher, B. K. Milthorpe, and M. A. Knackstedt, Assessment of bone ingrowth into porous biomaterials using micro-CT, Biomaterials 28, 2491 (2007).

[40] Q. Lin, B. Bijeljic, H. Rieke, and M. Blunt, Visualization and quantification of capillary drainage in the pore space of laminated sandstone by a porous plate method using differential imaging X-ray microtomography, Water Resour. Res. 53, 7457 (2017).

[41] M. Rücker, S. Berg, R. Armstrong, A. Georgiadis, H. Ott, L. Simon, F. Enzmann, M. Kersten, and S. de With, The fate of oil clusters during fractional flow: Trajectories in the saturation - capillary number space, in Conference Proceedings: International Symposium of the Society of Core Analysts, Vol. 7 (2015).

[42] R. Salathiel, Oil recovery by surface film drainage in mixedwettability rocks, J. Pet. Technol. 25, 1216 (1973).

[43] A. Q. Raeini, B. Bijeljic, and M. J. Blunt, Generalized network modeling: Network extraction as a coarse-scale discretization of the void space of porous media, Phys. Rev. E 96, 013312 (2017).

[44] G. Taubin, Curve and surface smoothing without shrinkage, in Proceedings of IEEE International Conference on Computer Vision (IEEE Computer Society Press, New York, 1995), pp. 852-857.
[45] N. R. Morrow, Physics and thermodynamics of capillary action in porous media, Ind. Eng. Chem. 62, 32 (1970).

[46] K. Culligan, D. Wildenschild, B. Christensen, W. Gray, and M. Rivers, Pore-scale characteristics of multiphase flow in porous media: A comparison of air-water and oil-water experiments, Adv. Water Resour. 29, 227 (2006).

[47] B. Raeesi, N. R. Morrow, and G. Mason, Capillary pressure hysteresis behavior of three sandstones measured with a multistep outflow-inflow apparatus, Vadose Zone J. 13 (2014), doi:10.2136/vzj2013.06.0097.

[48] P. H. Valvatne and M. J. Blunt, Predictive pore-scale modeling of two-phase flow in mixed wet media, Water Resour. Res. 40, W07406 (2004).

[49] K. Singh, B. Bijeljic, and M. J. Blunt, Imaging of oil layers, curvature and contact angle in a mixed-wet and a water-wet carbonate rock, Water Resour. Res. 52, 1716 (2016).

[50] M. Berger and B. Godtiaux, Differential Geometry: Manifolds, Curves, and Surfaces: Manifolds, Curves, and Surfaces (Springer Science \& Business Media, New York, 2012), Vol. 115.

[51] K. F. Gauss and P. Pesic, General Investigations of Curved Surfaces, Dover Books on Mathematics (Dover Publications, New York, 2005).

[52] W.-B. Bartels, H. Mahani, S. Berg, and S. Hassanizadeh, Literature review of low salinity waterflooding from a length and time scale perspective, Fuel 236, 338 (2019).

[53] S. Ljunggren and J. C. Eriksson, Minimal surfaces and Winsor III microemulsions, Langmuir 8, 1300 (1992).

[54] J. E. Taylor, The structure of singularities in soap-bubble-like and soap-film-like minimal surfaces, Ann. Math. 103, 489 (1976).

[55] G. Benedek, The structure of negatively curved spongy carbon, Diam. Relat. Mater. 12, 768 (2003).

[56] A. Mishra, V. D. Gordon, L. Yang, R. Coridan, and G. C. L. Wong, HIV TAT forms pores in membranes by inducing saddlesplay curvature: Potential role of bidentate hydrogen bonding, Angw. Chem. Intl. Ed. 47, 2986 (2008).

[57] M. J. Blunt, Q. Lin, T. Akai, and B. Bijeljic, A thermodynamically consistent characterization of wettability in porous media using high-resolution imaging, J. Colloid Interface Sci. 552, 59 (2019).

[58] A. AlRatrout, M. J. Blunt, and B. Bijeljic, Wettability in complex porous materials, the mixed-wet state, and its relationship to surface roughness, Proc. Natl. Acad. Sci. USA 115, 8901 (2018). 